\title{
Updated results of the OPERA long baseline neutrino experiment
}

\author{
S. Dusini ${ }^{* \dagger}$ \\ INFN - Padova \\ E-mail: stefano.dusiniepd.infn.it
}

The OPERA neutrino detector built in the underground Gran Sasso Laboratory is designed to detect muon-neutrino to tau-neutrino oscillations in direct appearance mode. The hybrid apparatus consists of an emulsion/lead target complemented by electronic detectors. It is placed in the long-baseline CERN to Gran Sasso neutrino beam (CNGS) $730 \mathrm{~km}$ away from the source. OPERA is taking data since 2008 and a first $v_{\tau}$ interaction candidate was published in 2010 base on a subsample of the data recorded in the first two year.

In this paper we present the results of the 2008-2009 events analysis together with the update of the OPERA sensitivity and background expectation base on the recent improvements in the analysis chain and in the Monte Carlo simulations.

The 2011 Europhysics Conference on High Energy Physics-HEP 2011,

July 21-27, 2011

Grenoble, Rhône-Alpes France

\footnotetext{
*Speaker.

${ }^{\dagger}$ On behalf of OPERA collaboration.
} 


\section{Introduction}

OPERA [1] is a long-baseline neutrino experiment designed to observe for the first time the $v_{\mu} \rightarrow v_{\tau}$ oscillation in direct appearance mode through the detection of the production of the corresponding $\tau$ lepton in the CNGS $v_{\mu}$ beam [2] over a baseline of $730 \mathrm{~km}$. OPERA is an hybrid detector consisting of two instrumented targets, each followed by a muon spectrometer. Each target is succession of walls of "ECC bricks". These are made of 561 -mm tick lead plates providing the mass interleaved with emulsion films with a micrometric resolution. For a detailed description of the OPERA detector we refer to [3]. The observation of a first $v_{\tau}$ candidate event in OPERA was reported in [4]. In this paper we report the results of the 2008 and 2009 events analysis highlighting the major improvements brought to the analysis chain and to the Monte Carlo simulations together with the updated OPERA sensitivity and background expectation.

\section{2008 and 2009 data analysis}

In the first two year of operation in the CNGS beam OPERA collect about $5.3 \times 10^{19}$ p.o.t. for a total of 5255 neutrino interactions reconstructed in the target. By the time of this conference $92 \%$ of the statistics was fully analyzed leading to 2738 events being located and fully reconstructed in the ECC brick. The fraction of located events with an identified muon in the final state is $81 \%$. The corresponding vertex location efficiencies are respectively $74 \pm 2 \%$ and $48 \pm 4 \%$ for Charged Current (CC) and Neutral Current (NC) $v_{\mu}$ interactions. After the neutrino interaction is located a scanning procedure is applied to all events to detect charged and neutral decays as well as secondary interaction and $\gamma$-ray conversion vertices in the vicinity of the primary vertex. When a secondary vertex is found, the kinematical analysis of the whole event is performed. The momentum of the particles not reaching the magnetic spectrometers is determined by multiple Coulomb scattering measured in the brick. The energy of $\gamma$-rays and electrons is estimated by a Neural Network algorithm based on the total track length and on the shape of the electromagnetic shower, together with the multiple Coulomb scattering of the leading tracks. This analysis procedure has lead to the observation so far of one $v_{\tau}$ candidate event in the $\tau \rightarrow h$ channel [4].

\begin{tabular}{|c|c|c|c|}
\hline Decay channel & \multicolumn{2}{|c|}{$\begin{array}{c}\text { Number of signal events expected for } \\
\end{array}$} & $\begin{array}{c}\text { Interaction vertex } \\
\text { location efficiency }\end{array}$ \\
\hline$\tau \rightarrow \mu$ & 1.79 & 0.39 & 0.54 \\
\hline$\tau \rightarrow e$ & 2.89 & 0.63 & 0.59 \\
\hline$\tau \rightarrow h$ & 2.25 & 0.49 & 0.59 \\
\hline$\tau \rightarrow 3 h$ & 0.71 & 0.15 & 0.64 \\
\hline Total & 7.63 & 1.65 & \\
\hline
\end{tabular}

Table 1: Expected numbers of observed signal events for $22.5 \times 10^{19}$ p.o.t. and for the analysed sample of the data accumulated in the 2008 and 2009 runs. Updated efficiencies for locating interaction vertices appear in the last column

The update of the expected numbers of events in the various $\tau$ channels for the nominal number of $22.5 \times 10^{19}$ p.o.t. and for the fraction of the 2008 and 2009 runs analysed at the time of this 
conference are shown in Table 1. Full mixing and $\Delta m_{23}^{2}=2.5 \times 10^{-3} \mathrm{eV}^{2}$ are assumed. The total number of signal events expected to be eventually detected has decreased from about 10 as quoted in the experiment proposal [1] to 8 , essentially caused by a reduction in the interaction vertex location efficiencies following our more reliable knowledge of the detector and of the analysis procedures which are now fully simulated in the emulsion off-line reconstruction software.

The main source of background to all $v_{\tau}$ signal channels is the production of charged charmed particles in $v_{\mu} \mathrm{CC}$ interactions where the primary muon is not identify and the charge of the opposite sign muon, if present, is not correctly measured. Using the OPERA electronic detectors $95 \%$ of the primary muons produced in charmed events are identified. To further increase the muon identification efficiency all the tracks at the primary interaction vertex of a signal candidate event emitted with a polar angle $\theta<1 \mathrm{rad}$ are followed in the bricks to search of possible interactions, scatterings or decays. About $30 \%$ of the muons not identified as such by the electronic detectors are recovered through the topology of their end-point, range-momentum correlation and energy loss measurement in the last fraction of their range. This technique allows also a reduction of the background in the $\tau \rightarrow \mu$ channel due to wrong associations of the muons emitted in $v_{\mu} \mathrm{CC}$ events to the vertex of secondary hadronic interactions with kink topologies. This is also true for fake muons in $\mathrm{NC}$ events.

The charm background has been evaluated using the recently published charm production cross-section [5]; they are significantly larger than those known at the time of the experiment proposal [1]. They lead to an increase of $35 \%$ of the charm production rate furthermore the relative charm fragmentation fraction into $D^{+}$has increased from $10 \%$ to $22 \%$. The overall effect is a charm background increase by a factor 1.6 to 2.4 depending on the decay channel.

\begin{tabular}{|c|c|c|c|c|c|c|c|c|}
\hline \multirow{2}{*}{$\begin{array}{c}\text { Decay } \\
\text { channel }\end{array}$} & \multicolumn{6}{|c|}{ Number of background events expected for } \\
& \multicolumn{5}{|c|}{$22.5 \times 10^{19}$ p.o.t. } & \multicolumn{5}{c|}{ Analysed sample } \\
& Charm & Hadron & Muon & Total & Charm & Hadron & Muon & Total \\
\hline$\tau \rightarrow \mu$ & 0.025 & 0.00 & 0.07 & $0.09 \pm 0.04$ & 0.00 & 0.00 & 0.02 & $0.02 \pm 0.01$ \\
\hline$\tau \rightarrow e$ & 0.22 & 0.00 & 0.00 & $0.22 \pm 0.05$ & 0.05 & 0.00 & 0.00 & $0.05 \pm 0.01$ \\
\hline$\tau \rightarrow h$ & 0.14 & 0.11 & 0.00 & $0.24 \pm 0.06$ & 0.03 & 0.02 & 0.00 & $0.05 \pm 0.01$ \\
\hline$\tau \rightarrow 3 h$ & 0.18 & 0.00 & 0.00 & $0.18 \pm 0.04$ & 0.04 & 0.00 & 0.00 & $0.04 \pm 0.01$ \\
\hline Total & 0.55 & 0.11 & 0.07 & $0.73 \pm 0.15$ & 0.12 & 0.02 & 0.02 & $0.16 \pm 0.03$ \\
\hline
\end{tabular}

Table 2: Expected numbers of observed background events from different sources for the nominal number of $22.5 \times 10^{19}$ p.o.t. and for the analysed sample of the data accumulated in the 2008 and 2009 runs. Errors quoted are systematic.

The second main background in the $\tau \rightarrow h$ decay channel come from one-prong inelastic interactions of primary hadrons produced in NC interactions, or in $\mathrm{CC}$ interactions where the primary lepton is not identified and in which no nuclear fragments can be associated with the secondary interaction. This has been evaluated with a FLUKA based Monte Carlo code, as detailed in [4] and cross-checked with OPERA and test beams data. Hadrons tracks from neutrino interactions in OPERA have been followed far from the primary vertex over a total length of $14 \mathrm{~m}$, equivalent to $2300 \mathrm{NC}$ events. No interactions have been found that fulfill the $\tau \rightarrow h$ selection criteria. Immediately outside the signal region, a total of 10 single-prong interactions have been observed with a 
$p_{T}$ larger than $200 \mathrm{MeV} / \mathrm{c}$, while 10.8 are expected from simulations. Bricks of the OPERA type have been also exposed to $4 \mathrm{GeV} / \mathrm{c} \pi^{-}$beams and about $190 \mathrm{~m}$ of track length have been followed searching for hadron interactions. The comparison of the data with FLUKA simulated data show a fair agreement.

The hadron interactions background can be further reduced by increasing the detection efficiency of highly ionizing particles, low energy protons and nuclear fragments. To increase the detection efficiency of those fragments at large angle, an image analysis tool has been developed where $2.5 \mathrm{~mm} \times 2.1 \mathrm{~mm} \times 24$ layers of high resolution microscope tomographic images are analysed in the upstream and downstream films of an interaction vertex. This technique has been applied to 64 interactions in an OPERA brick exposed to an $8 \mathrm{GeV} / \mathrm{c} \pi^{-}$beam. At least one highly ionising particle has been associated to $(56 \pm 7) \%$ of the events with a backward/forward asymmetry of $0.75 \pm 0.15$, while $53 \%$ are expected from simulations with an asymmetry of 0.71 . This technique provides an additional background reduction of about $20 \%$.

The update of the expected numbers of background events is reported in Table 2. Despite of the significant increase of charm cross section the total number of background events decrease by $2.7 \%$ with respect to [1] due to a significant improvement in the identification of the decay products as hadron or muon.

\section{Conclusions}

The OPERA experiment has completed the study of $92 \%$ of the data accumulated during the first two years of operation in the CNGS beam (2008-2009) with the aim of the first detection of neutrino oscillations in direct appearance mode. One $v_{\tau}$ candidate event has been observed in the $\tau \rightarrow h$ decay channel with an expectation of 1.65 signal events. The expected number background events in this channel is $0.05 \pm 0.01$ (syst.) leading to a significance of the observation of $95 \%$. Considering all decay channels, the numbers of expected background events is $0.16 \pm 0.03$ (syst.), the probability for the observed event to be background being $15 \%$.

\section{References}

[1] OPERA collaboration, M. Guler et al., An appearance experiment to search for $v_{\mu} \rightarrow v_{\tau}$ oscillations in the CNGS beam: experimental proposal, CERN-SPSC-2000-028, LNGS P25/2000. OPERA collaboration, M. Guler et al., Status Report on the OPERA experiment, CERN/SPSC 2001-025, LNGS-EXP 30/2001 add. 1/01.

[2] Ed. K. Elsener, The CERN Neutrino beam to Gran Sasso (Conceptual Technical Design), CERN 98-02, INFN/AE-98/05.

R. Bailey et al., The CERN Neutrino beam to Gran Sasso (NGS) (Addendum to report CERN 98-02, INFN/AE-98/05), CERN-SL/99-034(DI), INFN/AE-99/05.

[3] OPERA collaboration, R. Acquafredda et al., JINST 4 (2009) P04018.

[4] OPERA collaboration, N. Agafonova et al., Phys. Lett. B 691 (2010) 138.

[5] CHORUS collaboration, A. Kayis-Topaksu et al., CERN-PH-EP-2011-109, arXiv:1107.0613 [hep-ex], submitted to New J. Phys. 